\title{
On a functional equation related to a problem of G. Derfel
}

\section{MARIUSZ SUDZIK(D)}

Dedicated to Professor Karol Baron on the occasion of his 70th birthday.

\begin{abstract}
Two years ago, during the 21st European Conference on Iteration Theory, Gregory Derfel asked: "Does there exist a non-trivial bounded continuous solution of the equation $2 f(x)=f(x-1)+f(-2 x)$ ?" He repeated the question during the 55th International Symposium on Functional Equations. In this paper we present a partial solution of a more general problem, connected to the functional equation $f(x)=M\left(f\left(x+t_{1}\right), f\left(x+t_{2}\right), \ldots, f(x+\right.$ $\left.\left.t_{n-1}\right), f(a x)\right)$, where $n \in \mathbb{N}, t_{1}, t_{2}, \ldots, t_{n-1} \in \mathbb{R} \backslash\{0\}, a \in(-\infty, 0)$ and $M$ is a given function in $n$ variables satisfying some additional properties. In particular, $M$ can be a weighted quasi-arithmetic mean in $n$ variables.
\end{abstract}

Mathematics Subject Classification. Primary 39B22; Secondary 39B05.

Keywords. Archetypal equation, Quasi-arithmetic mean, Functional equations, Equations with rescaling.

\section{Introduction}

In $[2,3]$ Bogachev, Derfel and Molchanov considered the functional equation

$$
f(x)=\iint_{\mathbb{R}^{2}} f(a(x-b)) d \mu(a, b),
$$

where $\mu$ is a given probability measure on $\mathbb{R}^{2}$. The study of Eq. (1.1) was initiated by Gregory Derfel in 1989 (see [5]). A large number of interesting results connected to (1.1), its particular cases and equations of a similar form can be found in $[1,6]$.

In this place it is worth mentioning that one can see Eq. (1.1) in terms of random variables. Indeed, let $(\Omega, \mathcal{A}, P)$ be a fixed probability space and assume that $(\alpha, \beta): \Omega \rightarrow \mathbb{R}^{2}$ is a random vector whose distribution is equal to $\mu$. Then equation

$$
f(x)=\int_{\Omega} f(\alpha(\omega)(x-\beta(\omega))) d P(\omega)
$$

is equivalent to (1.1). 
Equation (1.1) was called archetypal equation in [2,3] since it is a rich source of many famous functional and differential-functional equations. For example Derfel showed in [5] that one can obtain many variants of the pantograph equation from (1.1). For details and examples the reader is referred to $[2,3]$. Notice that, clearly, every constant function satisfies (1.1); these are trivial solutions. The authors asked there if Eq. (1.1) has a non-trivial bounded continuous solution. The class of bounded and continuous functions is considered because of an important role played by this class in applications (for example in astrophysics and risk theory). The interested reader is referred to [3].

The authors answered the problem of the existence of non-constant bounded continuous solutions of (1.1) for a large class of probability measures (see $[2,3])$. The behaviour of solutions depends especially on the value of $K:=$ $\iint_{\mathbb{R}^{2}} \ln |a| d \mu(a, b)$, which was shown by Derfel in [5]. An influence of this number on (1.1) can be exemplified by the following result.

[3, Th. 1.1] Assume that $\iint_{\mathbb{R}^{2}} \ln (\max (|b|, 1)) d \mu(a, b)$ and $K$ are finite and suppose that $P(\alpha=0)=0, P(|\alpha|=1)<1$ and $P(\alpha(c-\beta)=c))<1$ for every $c \in \mathbb{R}$.

(i) If $K<0$, then any bounded continuous solution of (1.1) is constant.

(ii) If $K>0$ and $\alpha>0$ a.s., then there is a non-constant bounded continuous solution of (1.1).

Non-constant solutions which are constructed in $(i i)$ are based on the theorems discovered by Grintsevichyus (see [7]). It should be added that the assumption " $\alpha>0$ a.s." is necessary. Under stronger regularity conditions, namely absolute continuity with a Lebesgue integrable derivative, there are no non-constant solutions of the archetypal equation in the case when $P(\alpha<$ $0)>0$ (see Theorem 4.5 in [2]). Unfortunately, we still do not have a wide knowledge about the existence of non-trivial bounded continuous solutions of (1.1) for $K>0$ and $P(\alpha<0)>0$. It is worth noting that when $P(\alpha<0)>0$ we are able to obtain (and, moreover, describe all) non-constant bounded continuous solutions only if $P(\alpha=-1)>0$ and $|\alpha|=1$ a.s. (see Theorem 2.3 in [3]); each of which is uniformly continuous.

On this account Derfel proposed to consider some special version of Eq. (1.1) with particular coefficients. During the 21st European Conference on Iteration Theory, held in Innsbruck, Austria, in 2016 he posed the following

Problem. Does there exist a non-constant bounded continuous solution $f: \mathbb{R} \rightarrow \mathbb{R}$ of the equation

$$
f(x)=\frac{1}{2} f(x-1)+\frac{1}{2} f(-2 x) ?
$$

The question was repeated by him a year later, during the 55th International Symposium on Functional Equations held in Chengdu, China.

The main result of this paper (Theorem 3.5) asserts in particular that every bounded continuous solution of Eq. (1.3), reaching its global extremum, 
is constant. In fact this theorem holds for Eq. (3.1) which is considerably more general than (1.3). In the last part we discuss the case when a solution does not attain the global extreme value.

\section{Preliminaries}

In what follows we fix a postive integer $n \geq 2$, a negative $a \in \mathbb{R}$, non-zero real numbers $t_{1}, \ldots, t_{n-1}$ and $\alpha, \beta \in \mathbb{R}$ such that $\alpha<\beta$. Let $M:[\alpha, \beta]^{n} \rightarrow[\alpha, \beta]$ be an arbitrary function.

We consider the following hypotheses $\left(A_{1}\right)-\left(A_{4}\right)$ :

$\left(A_{1}\right) \quad$ The function $M(\cdot, \ldots, \cdot, \alpha)$ is continuous with respect to each variable.

$\left(A_{2}\right) \quad$ The function $M(\cdot, \ldots, \cdot, \alpha)$ is strictly increasing with respect to each variable.

$\left(A_{3}\right) \quad M\left(x_{1}, \ldots, x_{n}\right)=\alpha \Longleftrightarrow x_{1}=\cdots=x_{n}=\alpha \quad$ for all $x_{1}, \ldots, x_{n} \in[\alpha, \beta]$.

$\left(A_{4}\right) \quad M(x, \ldots, x, \alpha) \neq x \quad$ for all $x \in(\alpha, \beta]$.

We also introduce the dual hypotheses:

$\left(B_{1}\right) \quad$ The function $M(\cdot, \ldots, \cdot, \beta)$ is continuous with respect to each variable.

$\left(B_{2}\right) \quad$ The function $M(\cdot, \ldots, \cdot, \beta)$ is strictly increasing with respect to each variable.

$\left(B_{3}\right) \quad M\left(x_{1}, \ldots, x_{n}\right)=\beta \Longleftrightarrow x_{1}=\cdots=x_{n}=\beta \quad$ for all $x_{1}, \ldots, x_{n} \in[\alpha, \beta]$.

$\left(B_{4}\right) \quad M(x, \ldots, x, \beta) \neq x \quad$ for all $x \in[\alpha, \beta)$.

Remark 2.1. According to the well-known "folk" theorem if $M:[\alpha, \beta]^{n} \rightarrow$ $[\alpha, \beta]$ satisfies hypotheses $\left(A_{1}\right)-\left(A_{2}\right)$ or $\left(B_{1}\right)-\left(B_{2}\right)$, then the function $M(\cdot, \ldots, \cdot, \alpha)$ is continuous.

There exists a very wide and natural class of functions satisfying all the above hypotheses, namely that of means introduced by J. Matkowski (see [9]) and shortly described below, which contains a smaller but better known class of weighted quasi-arithmetic means.

Let $I \subseteq \mathbb{R}$ be an interval. Recall that a function $M: I^{n} \rightarrow \mathbb{R}$ is called a mean on $I$ if

$$
\min \left\{x_{1}, x_{2}, \ldots, x_{n}\right\} \leq M\left(x_{1}, x_{2}, \ldots, x_{n}\right) \leq \max \left\{x_{1}, x_{2}, \ldots, x_{n}\right\}
$$

for all $x_{1}, x_{2}, \ldots, x_{n} \in I$. Observe that, in fact, $M$ maps the cube $I^{n}$ onto the interval $I$. The mean $M$ is called strict if the above inequalities are sharp whenever $x_{i} \neq x_{j}$ for some $i, j \in\{1,2, \ldots, n\}$.

Hence, we can deduce the following. 
Remark 2.2. If $M$ is a continuous mean, strictly increasing with respect to each variable, then $M$ satisfies hypotheses $\left(A_{1}\right)-\left(A_{4}\right)$ and $\left(B_{1}\right)-\left(B_{4}\right)$.

Let $I \subseteq \mathbb{R}$ be an interval and $\varphi_{1}, \varphi_{2}, \ldots, \varphi_{n}: I \rightarrow \mathbb{R}$ be continuous strictly monotonic functions of the same type of monotonicity. Then the function $M$ : $I^{n} \rightarrow \mathbb{R}$, defined by

$$
M\left(x_{1}, \ldots, x_{n}\right)=\left(\varphi_{1}+\cdots+\varphi_{n}\right)^{-1}\left(\varphi_{1}\left(x_{1}\right)+\cdots+\varphi_{n}\left(x_{n}\right)\right),
$$

is a mean on $I$ called the Matkowski mean generated by $\varphi_{1}, \ldots, \varphi_{n}$ (see [9]).

Remark 2.3. Every Matkowski mean $M:[\alpha, \beta]^{n} \rightarrow \mathbb{R}$ is strictly increasing with respect to each variable and continuous, so it serves as an example of functions satisfying hypotheses $\left(A_{1}\right)-\left(A_{4}\right)$ and $\left(B_{1}\right)-\left(B_{4}\right)$.

At the end of this chapter let us note the following simple observation.

Remark 2.4. Let $i \in\{1,2,3,4\}$ be fixed. If $M:[\alpha, \beta]^{n} \rightarrow[\alpha, \beta]$ satisfies hypothesis $B_{i}$, then the function $N:[-\beta,-\alpha]^{n} \rightarrow[-\beta,-\alpha]$, defined by

$$
N\left(x_{1}, x_{2}, \ldots, x_{n}\right)=-M\left(-x_{1},-x_{2}, \ldots,-x_{n}\right),
$$

satisfies hypothesis $A_{i}$ with the parameters $-\beta$ and $-\alpha$.

\section{The main theorem}

This section concerns functions $f: \mathbb{R} \rightarrow \mathbb{R}$ satisfying the functional equation

$$
f(x)=M\left(f\left(x+t_{1}\right), f\left(x+t_{2}\right), \ldots, f\left(x+t_{n-1}\right), f(a x)\right) .
$$

Equation (1.3) is a particular case of Eq. (3.1): if we put $n=2, t_{1}=-1$, $a=-2$ and let $M$ be the arithmetic mean, then (3.1) takes the form (1.3). Moreover, $M$ satisfies hypotheses $\left(A_{1}\right)-\left(A_{4}\right)$ and $\left(B_{1}\right)-\left(B_{4}\right)$ with arbitrary $\alpha, \beta \in \mathbb{R}$ such that $\alpha<\beta$.

In this chapter we consider the functions reaching the global extreme value. We start with a very simple observation.

Remark 3.1. Let $f: \mathbb{R} \rightarrow[\alpha, \beta]$ be a solution of Eq. (3.1) reaching the global maximum. Then the function $g:=-f$ reaches the global minimum and satisfies the equation

$$
g(x)=N\left(g\left(x+t_{1}\right), g\left(x+t_{2}\right), \ldots, g\left(x+t_{n-1}\right), g(a x)\right)
$$

with $N$ given by formula (2.1).

The following two possibilities will be covered by our results:

(a) $M$ satisfies $\left(A_{1}\right)-\left(A_{4}\right)$ and $f$ takes its global minimum,

(b) $M$ satisfies $\left(B_{1}\right)-\left(B_{4}\right)$ and $f$ takes its global maximum. 
Results in this chapter are formulated and proved for the first case only. The second case can be obtained by a similar argumentation or applying Remarks 2.3 and 3.1. In particular, the results presented below hold when $M$ is a Matkowski mean.

We are going to prove two useful lemmas.

Lemma 3.2. Assume that $M$ satisfies hypotheses $\left(A_{1}\right),\left(A_{2}\right)$ and $\left(A_{4}\right)$. Then the sequence $\left(u_{k}\right)_{k \in \mathbb{N}_{0}}$, defined by $u_{0}=\beta$ and

$$
u_{k}=M\left(u_{k-1}, u_{k-1}, \ldots, u_{k-1}, \alpha\right) \quad \text { for } k \in \mathbb{N},
$$

is strictly decreasing and converges to $\alpha$.

Proof. Observe that by $\left(A_{4}\right)$ we have $u_{1} \neq \beta$, and thus $u_{1}<u_{0}$. Assume that for some $k \in \mathbb{N}$ we have $u_{k}<u_{k-1}$. Using $\left(A_{2}\right)$ we get

$$
u_{k+1}=M\left(u_{k}, u_{k}, \ldots, u_{k}, \alpha\right)<M\left(u_{k-1}, u_{k-1}, \ldots, u_{k-1}, \alpha\right)=u_{k} .
$$

Therefore the sequence $\left(u_{k}\right)_{k \in \mathbb{N}_{0}}$ is strictly decreasing. We also know that $\left(u_{k}\right)_{k \in \mathbb{N}_{0}}$ is bounded, and thus convergent. Let

$$
u_{\infty}:=\lim _{k \rightarrow \infty} u_{k}
$$

Note that, by $\left(A_{1}\right)$

$$
u_{\infty}=\lim _{k \rightarrow \infty} M\left(u_{k}, u_{k}, \ldots, u_{k}, \alpha\right)=M\left(u_{\infty}, u_{\infty}, \ldots, u_{\infty}, \alpha\right)
$$

and now hypothesis $\left(A_{4}\right)$ implies that $u_{\infty}=\alpha$.

Lemma 3.3. Assume that $M$ satisfies hypothesis $\left(A_{3}\right)$ and let $f: \mathbb{R} \rightarrow[\alpha, \beta]$ be a solution of Eq. (3.1). If there exists $x_{0} \in \mathbb{R}$ such that $f\left(x_{0}\right)=\alpha$, then

$$
f\left(x_{0}+\sum_{i=1}^{n-1} k_{i} t_{i}\right)=\alpha
$$

for all $k_{1}, k_{2}, \ldots, k_{n-1} \in \mathbb{N}_{0}$.

Proof. Fix arbitrary $k_{1}, k_{2}, \ldots, k_{n-1} \in \mathbb{N}_{0}$. Applying equality (3.1) to $x=x_{0}$ we get

$$
\alpha=f\left(x_{0}\right)=M\left(f\left(x_{0}+t_{1}\right), f\left(x_{0}+t_{2}\right), \ldots, f\left(x_{0}+t_{n-1}\right), f\left(a x_{0}\right)\right) .
$$

Now $\left(A_{3}\right)$ implies that

$$
f\left(x_{0}+t_{i}\right)=\alpha \quad \text { for all } i=1,2, \ldots, n-1 .
$$

Repeating this reasoning $k_{i}$ times for all $i=1,2, \ldots, n-1$ we come to the required equality. 
Corollary 3.4. Assume that $M$ satisfies hypothesis $\left(A_{3}\right)$ and let $f: \mathbb{R} \rightarrow[\alpha, \beta]$ be a solution of Eq. (3.1). If there exists $x_{0} \in \mathbb{R}$ such that $f\left(x_{0}\right)=\alpha$, then

$$
f\left(a x_{0}+\sum_{i=1}^{n-1}\left(l_{i}+a k_{i}\right) t_{i}\right)=\alpha
$$

for all $k_{1}, k_{2}, \ldots, k_{n-1}, l_{1}, l_{2}, \ldots, l_{n-1} \in \mathbb{N}_{0}$.

Proof. Fix $k_{1}, \ldots, k_{n-1}, l_{1}, \ldots, l_{n-1} \in \mathbb{N}_{0}$. If we apply Lemma 3.3, we obtain

$$
f\left(x_{0}+\sum_{i=1}^{n-1} k_{i} t_{i}\right)=\alpha .
$$

In the next step we can apply equality (3.1) to the point $x_{0}+\sum_{i=1}^{n-1} k_{i} t_{i}$. Making use of $\left(A_{3}\right)$ we get

$$
f\left(a x_{0}+\sum_{i=1}^{n-1} a k_{i} t_{i}\right)=\alpha .
$$

Now, applying Lemma 3.3 to the point $a x_{0}+\sum_{i=1}^{n-1} a k_{i} t_{i}$, we come to

$$
f\left(a x_{0}+\sum_{i=1}^{n-1}\left(l_{i}+a k_{i}\right) t_{i}\right)=f\left(a x_{0}+\sum_{i=1}^{n-1} l_{i} t_{i}+\sum_{i=1}^{n-1} a k_{i} t_{i}\right)=\alpha .
$$

Now we are in a position to prove the main theorem.

Theorem 3.5. Assume that $a \neq-1, M$ satisfies hypotheses $\left(A_{1}\right)-\left(A_{4}\right)$ and let $f: \mathbb{R} \rightarrow[\alpha, \beta]$ be a continuous solution of Eq. (3.1). If $f$ attains the global minimum, then $f$ is constant.

Proof. Let $x_{0} \in \mathbb{R}$ be such that $f\left(x_{0}\right)=\alpha$. The proof depends on the parameter $a$, so it is split into three cases.

Case 1: $a \in \mathbb{Z}$. We define the sequence $\left(D_{j}\right)_{j \in \mathbb{N}_{0}}$ of sets setting

$$
D_{j}:=\left\{\frac{a x_{0}+\sum_{i=1}^{n-1} s_{i} t_{i}}{a^{j}}: s_{1}, \ldots, s_{n-1} \in \mathbb{Z}\right\}
$$

and let

$$
D:=\bigcup_{j=0}^{\infty} D_{j} .
$$

Since $a<-1$, the set $D$ is dense in $\mathbb{R}$. The sequence $\left(D_{j}\right)_{j \in \mathbb{N}_{0}}$ also has the following properties:

$$
\begin{array}{ll}
D_{j}+t_{i} \subset D_{j} & \text { for all } j \in \mathbb{N}_{0} \text { and } i=1,2, \ldots, n-1, \\
a D_{j+1} \subset D_{j} & \text { for all } j \in \mathbb{N}_{0} .
\end{array}
$$


By Corollary 3.4 we have

$$
f\left(a x_{0}+\sum_{i=1}^{n-1}\left(l_{i}+a k_{i}\right) t_{i}\right)=\alpha \quad \text { for all } k_{1}, \ldots, k_{n-1}, l_{1}, \ldots, l_{n-1} \in \mathbb{N}_{0}
$$

Since $a$ is a negative integer, the expression $l_{i}+a k_{i}$ runs through the whole set $\mathbb{Z}$ while $i=1,2, \ldots, n-1$. Therefore

$$
f\left(a x_{0}+\sum_{i=1}^{n-1} s_{i} t_{i}\right)=\alpha \quad \text { for every } s_{1}, s_{2}, \ldots, s_{n-1} \in \mathbb{Z} .
$$

This means that $f_{\mid D_{0}}=\alpha$. Assume that $f_{\mid D_{j}}=\alpha$ for a $j \in \mathbb{N}_{0}$ and let $x \in D_{j+1}$ be fixed. We show that $f(x)=\alpha$. Let $\left(u_{k}\right)_{k \in \mathbb{N}_{0}}$ be the sequence defined by (3.2) and let $y=x+\sum_{i=1}^{n-1} k_{i} t_{i}$, where $k_{1}, k_{2}, \ldots, k_{n-1} \in \mathbb{N}_{0}$. Then $f(y) \leq \beta=u_{0}$. Assume that for some $p \in \mathbb{N}_{0}$ we have

$$
f\left(x+\sum_{i=1}^{n-1} k_{i} t_{i}\right) \leq u_{p} \quad \text { for all } k_{1}, k_{2}, \ldots, k_{n-1} \in \mathbb{N}_{0}
$$

Take $y=x+\sum_{i=1}^{n-1} k_{i} t_{i}$ for some $k_{1}, k_{2}, \ldots, k_{n-1} \in \mathbb{N}_{0}$. Then, by (3.3) and (3.4), we have $a y \in D_{j}$. Therefore, using condition (3.5) and hypothesis $\left(A_{2}\right)$, we come to

$$
\begin{aligned}
f(y) & =M\left(f\left(y+t_{1}\right), f\left(y+t_{2}\right), \ldots, f\left(y+t_{n-1}\right), f(a y)\right) \\
& =M\left(f\left(y+t_{1}\right), f\left(y+t_{2}\right), \ldots, f\left(y+t_{n-1}\right), \alpha\right) \\
& \leq M\left(u_{p}, u_{p}, \ldots, u_{p}, \alpha\right)=u_{p+1} .
\end{aligned}
$$

Thus, using induction, we have

$$
f(x) \leq u_{p} \text { for every } p \in \mathbb{N}_{0},
$$

hence, by Lemma 3.2,

$$
f(x) \leq \alpha
$$

Thus $f_{\mid D_{j+1}}=\alpha$ because the point $x$ was arbitrary. Consequently,

$$
f(x)=\alpha \text { for every } x \in D .
$$

In this case the proof is finished because of the continuity of $f$ and the density of the set $D$.

Case 2: $a \in \mathbb{Q} \backslash \mathbb{Z}$. Choose $p \in \mathbb{Z}$ and $q \in \mathbb{N}$ such that $a=p / q$ and $p, q$ are coprime. Then obviously $p<0$ and $q \geq 2$. By Corollary 3.4 we have

$$
f\left(a x_{0}+\sum_{i=1}^{n-1}\left(k_{i}+\frac{p l_{i}}{q}\right) t_{i}\right)=\alpha
$$


for every $k_{1}, \ldots, k_{n-1}, l_{1}, \ldots, l_{n-1} \in \mathbb{N}_{0}$. The expression $k_{i}+p l_{i} / q$ runs through the set $\mathbb{Z} / q$ while $i=1,2, \ldots, n-1$. Hence we have

$$
f\left(a x_{0}+\frac{\sum_{i=1}^{n-1} s_{i} t_{i}}{q}\right)=\alpha \quad \text { for every } s_{1}, \ldots, s_{n-1} \in \mathbb{Z} .
$$

Now we define a sequence $\left(D_{j}\right)_{j \in \mathbb{N}}$ of sets putting

$$
D_{j}:=\left\{a^{j} x_{0}+\frac{\sum_{i=1}^{n-1} s_{i} t_{i}}{q^{j}}: s_{1}, \ldots, s_{n-1} \in \mathbb{Z}\right\}
$$

and letting

$$
D:=\bigcup_{j=1}^{\infty} D_{j} .
$$

The set $D$ is dense in $\mathbb{R}$ as $q>1$. The equality $f_{\mid D_{1}}=\alpha$ was shown above. Assume that $f_{\mid D_{j}}=\alpha$ for some $j \in \mathbb{N}$. Let $x \in D_{j}$ be fixed. Then there exist $s_{1}, \ldots, s_{n-1} \in \mathbb{Z}$ such that $x=a^{j} x_{0}+\sum_{i=1}^{n-1} s_{i} t_{i} / q^{j}$. If we apply equality (3.1) to the point $x$, we get

$$
\alpha=f(x)=M\left(f\left(x+t_{1}\right), \ldots, f\left(x+t_{n-1}\right), f(a x)\right) .
$$

Hence, by hypothesis $\left(A_{3}\right)$, we obtain $f(a x)=\alpha$. This equality means that

$$
f\left(a^{j+1} x_{0}+\frac{\sum_{i=1}^{n-1} p s_{i} t_{i}}{q^{j+1}}\right)=\alpha .
$$

Applying Lemma 3.3 to the point $a^{j+1} x_{0}+\sum_{i=1}^{n-1} p s_{i} t_{i} / q^{j+1}$, we obtain

$$
f\left(a^{j+1} x_{0}+\sum_{i=1}^{n-1} \frac{q^{j+1} k_{i}+p s_{i}}{q^{j+1}} t_{i}\right)=\alpha \quad \text { for all } k_{1}, \ldots, k_{n-1} \in \mathbb{N}_{0} .
$$

The point $x$ was taken arbitrarily. Therefore we have proved that $f$ takes the value $\alpha$ on the set

$$
\left\{a^{j+1} x_{0}+\frac{\sum_{i=1}^{n-1}\left(q^{j+1} k_{i}+p s_{i}\right) t_{i}}{q^{j+1}}: s_{1}, \ldots, s_{n-1} \in \mathbb{Z}, k_{1}, \ldots, k_{n-1} \in \mathbb{N}_{0}\right\} \text {. }
$$

Since $q^{j+1}$ and $p$ are coprime, the expression $q^{j+1} k_{i}+p s_{i}$ runs through the set $\mathbb{Z}$ while $i=1, \ldots, n-1$. Consequently, the set just above is equal to $D_{j+1}$. Hence, by induction, we get $f_{\mid D}=\alpha$ and the proof is completed as $D$ is a dense subset of the real line and $f$ is continuous.

Case 3: $a \in(-\infty, 0) \backslash \mathbb{Q}$. By Corollary 3.4 we have

$$
f\left(a x_{0}+(l+a k) t_{1}\right)=\alpha \text { for every } k, l \in \mathbb{N}_{0} .
$$

Kronecker's density theorem (see Chapter XXIII in [8]) asserts that the set $D:=\left\{l+a k: l, k \in \mathbb{N}_{0}\right\}$ is dense in $\mathbb{R}$. Therefore also the set $a x_{0}+D t_{1}$ is dense in $\mathbb{R}$ and thus $f$ is constant. 
Remark 3.6. Note that hypotheses $\left(A_{1}\right),\left(A_{2}\right)$ and $\left(A_{4}\right)$ are important only in the first case of the proof. In the case when $a \notin \mathbb{Z}$ hypotheses $\left(A_{1}\right),\left(A_{2}\right)$ and $\left(A_{4}\right)$ can be ommited.

Remark 3.7. Note that if $M$ is a weighted arithmetic mean and $|a|<1$, then every bounded continuous solution of Eq. (3.1) is constant. This follows as a special case of Theorem 3.2 from [2], with no additional assumptions on $f$.

Remark 3.8. Theorem 3.5 provides a partial answer to the problem of Gregory Derfel:

If $f: \mathbb{R} \rightarrow \mathbb{R}$ is a bounded continuous solution of Eq. (1.3) reaching the global minimum (maximum), then $f$ is constant.

\section{Further results}

The situation when $a=-1$ is completely different. Now Eq. (3.1) takes the form

$$
f(x)=M\left(f\left(x+t_{1}\right), f\left(x+t_{2}\right), \ldots, f\left(x+t_{n-1}\right), f(-x)\right) .
$$

The following obvious fact shows that Eq. (4.1) can have a great number of non-constant solutions belonging to the class of bounded continuous functions and reaching the global minimum and global maximum.

Remark 4.1. If $M$ is reflexive, i.e.

$$
M(x, x, x, \ldots, x)=x \text { for every } x \in[\alpha, \beta],
$$

and there exists $s \in \mathbb{R} \backslash\{0\}$ such that all fractions $\frac{t_{1}}{s}, \frac{t_{2}}{s}, \ldots, \frac{t_{n-1}}{s}$ are integers, then every continuous even function $f: \mathbb{R} \rightarrow \mathbb{R}$, with period $s$, is a bounded solution of Eq. (4.1), attaining both of its global extremes.

If such $s$ cannot be found, we only have the trivial solutions.

Remark 4.2. Assume that $M$ satisfies hypothesis $\left(A_{3}\right)\left[\left(B_{3}\right)\right]$ and there are numbers $p, q \in\{1,2, \ldots, n-1\}$ such that $\frac{t_{p}}{t_{q}} \notin \mathbb{Q}$ and let $f: \mathbb{R} \rightarrow[\alpha, \beta]$ be a bounded continuous solution of Eq. (4.1). If $f$ attains the global minimum [maximum], then $f$ is constant.

Proof. Let $x_{0} \in \mathbb{R}$ be such that $f\left(x_{0}\right)=\alpha$. By Corollary 3.4 we have

$$
f\left(-x_{0}+\left(k_{p}-l_{p}\right) t_{p}+\left(k_{q}-l_{q}\right) t_{q}\right)=\alpha
$$

for every $k_{p}, k_{q}, l_{p}, l_{q} \in \mathbb{N}_{0}$, or simply

$$
f\left(-x_{0}+s_{p} t_{p}+s_{q} t_{q}\right)=\alpha \quad \text { for all } s_{p}, s_{q} \in \mathbb{Z} .
$$


By Kronecker's density theorem (see [8]) we know that the set $\left\{s_{q}+s_{p} t_{p} / t_{q}\right.$ : $\left.s_{p}, s_{q} \in \mathbb{Z}\right\}$ is dense in the real line. Consequently, also $\left\{s_{p} t_{p}+s_{q} t_{q}-x_{0}\right.$ : $\left.s_{p}, s_{q} \in \mathbb{Z}\right\}$ is a dense subset of $\mathbb{R}$, and thus $f$ is constant.

In the case when $M$ is a weighted arithmetic mean, Remark 4.1, its converse and Remark 4.2 are special cases of Theorem 2.3 in [3], which is an extension of the Choquet-Deny theorem (see [4]).

If a bounded continuous solution of (3.1) does not attain the global minimum or maximum, then the problem of its form is much more difficult and is still unsolved. The result below (compare with Theorems 4.2 and 4.3 in [2]) describes some properties of possible non-constant solutions of Eq. (3.1).

Proposition 4.3. Let $M$ be a continuous function satisfying hypothesis $\left(A_{3}\right)$ $\left[\left(B_{3}\right)\right]$ and let $f: \mathbb{R} \rightarrow \mathbb{R}$ be a continuous solution of Eq. (3.1) such that $\inf f(\mathbb{R})=\alpha[\sup f(\mathbb{R})=\beta]$. If

$$
\alpha<f(x)<\beta \quad \text { for every } x \in \mathbb{R},
$$

then for all $c \in \mathbb{R}$

$$
\begin{gathered}
\alpha=\inf \{f(x): x>c\}=\inf \{f(x): x<c\} \\
{[\beta=\sup \{f(x): x>c\}=\sup \{f(x): c<x\}] .}
\end{gathered}
$$

Proof. We prove this theorem under the assumptions $\left(A_{3}\right)$ and $\inf f(\mathbb{R})=\alpha$ only. Let $c \in \mathbb{R}$ and take any sequence $\left(x_{n}\right)_{n \in \mathbb{N}}$ of real numbers such that

$$
\lim _{n \rightarrow \infty} f\left(x_{n}\right)=\alpha \text {. }
$$

If it was bounded, then one could find a subsequence of $\left(x_{n}\right)_{n \in \mathbb{N}}$ convergent to some point $x_{0} \in \mathbb{R}$. Hence, by the continuity of $f$, we would get $f\left(x_{0}\right)=\alpha$, a contradiction. Therefore $\left(x_{n}\right)_{n \in \mathbb{N}}$ is unbounded. Assume for instance that it is unbounded from above. Then the interval $(c,+\infty)$ contains infinitely many elements of this sequence. Let $\left(x_{n_{k}}\right)_{k \in \mathbb{N}}$ be its subsequence such that $x_{n_{k}}>c$ for every $k \in \mathbb{N}$. Since $f\left(x_{n}\right) \rightarrow \alpha$, we have

$$
\lim _{k \rightarrow \infty} f\left(x_{n_{k}}\right)=\alpha
$$

and thus

$$
\alpha=\inf \{f(x): x>c\} .
$$

It remains to show that $\alpha=\inf \{f(x): x<c\}$. Suppose that the sequence $\left(f\left(a x_{n_{k}}\right)\right)_{k \in \mathbb{N}}$ is not convergent to $\alpha$. The sequences $\left(f\left(a x_{n_{k}}\right)\right)_{k \in \mathbb{N}}$ and $\left(f\left(x_{n_{k}}+\right.\right.$ $\left.\left.t_{i}\right)\right)_{k \in \mathbb{N}}$ are bounded for each $i=1, \ldots, n-1$. We can choose a subsequence $\left(x_{n_{k_{l}}}\right)_{l \in \mathbb{N}}$ of the sequence $\left(x_{n_{k}}\right)_{k \in \mathbb{N}}$ such that the sequences $\left(f\left(x_{n_{k_{l}}}+t_{i}\right)\right)_{l \in \mathbb{N}}$ are convergent for every $i \in\{1,2, \ldots, n-1\}$ and the sequence $\left(f\left(a x_{n_{k_{l}}}\right)\right)_{l \in \mathbb{N}}$ 
has a limit different from $\alpha$. Then, by the continuity of $M$ and hypothesis $\left(A_{3}\right)$, we get

$$
\lim _{l \rightarrow \infty} M\left(f\left(x_{n_{k_{l}}}+t_{1}\right), f\left(x_{n_{k_{l}}}+t_{2}\right), \ldots, f\left(x_{n_{k_{l}}}+t_{n-1}\right), f\left(a x_{n_{k_{l}}}\right)\right)>\alpha .
$$

On the other hand $f\left(x_{n_{k_{l}}}\right) \rightarrow \alpha$. It contradicts the assumption that $f$ is a solution of Eq. (3.1). Therefore $f\left(a x_{n_{k}}\right) \rightarrow \alpha$ and there exists $k_{0} \in \mathbb{N}$ such that $a x_{n_{k}}<c$ for every $k \geq k_{0}$. Hence inf $\{f(x): x<c\}=\alpha$.

We immediately get the following

Corollary 4.4. Let $M$ be a continuous function satisfying hypotheses $\left(A_{3}\right)$ and $\left(B_{3}\right)$ and let $f: \mathbb{R} \rightarrow \mathbb{R}$ be a continuous solution of Eq. (3.1) such that $\inf f(\mathbb{R})=\alpha$ and $\sup f(\mathbb{R})=\beta$. If

$$
\alpha<f(x)<\beta \quad \text { for every } x \in \mathbb{R},
$$

then $f$ has no horyzontal asymptotes.

In particular the meaning of these theorems is that every non-constant solution of Eq. (1.3) should be oscillating in infinity. So the main question is about the existence or non-existence of such oscillating solutions.

Open Access. This article is distributed under the terms of the Creative Commons Attribution 4.0 International License (http://creativecommons.org/licenses/by/4.0/), which permits unrestricted use, distribution, and reproduction in any medium, provided you give appropriate credit to the original author(s) and the source, provide a link to the Creative Commons license, and indicate if changes were made.

\section{References}

[1] Baron, K., Jarczyk, W.: Recent results on functional equations in a single variable, perspectives and open problems. Aequ. Math. 61, 1-48 (2001)

[2] Bogachev, L.V., Derfel, G., Molchanov, S.A.: Analysis of the archetypal functional equation in the non-critical case. In: AIMS Proceedings, Springfield, pp. 132-141 (2015)

[3] Bogachev, L.V., Derfel, G., Molchanov, S.A.: On bounded continuous solutions of the archetypal equation with rescaling. Proc. R. Soc. A471, 1-19 (2015)

[4] Choquet, G., Deny, J.: Sur l'équation de convolution $\mu=\mu \star \sigma$ (French) [On the convolution equation $\mu=\mu \star \sigma]$. C. R. Acad. Sci. Paris 250, 799-801 (1960)

[5] Derfel, G.: Probabilistic method for a class of functional-differential equations. Ukr. Math. J. 41, 1137-1141 (1989)

[6] Derfel, G.: Functional-differential and functional equations with rescaling. In: Gohberg, I., Langer, H. (eds.) Operator theory and boundary eigenvalue problems. Operator Theory: Advances and Applications, vol. 80, pp. 100-111. Birkhuser, Basel, Switzerland (1995)

[7] Grintsevichyus, A.K.: On the continuity of the distribution of a sum of dependent variables connected with independent walks on lines. Theor. Probab. Appl. 19, 163-168 (1974)

[8] Hardy, G.H., Wright, E.M.: An Introduction to the Theory of Numbers. Oxford University Press, London (1960)

[9] Matkowski, J.: Generalized weighted and quasi-arithmetic means. Aequ. Math. 79(3), 203-212 (2010) 
Mariusz Sudzik

Faculty of Mathematics, Computer Science and Econometrics

University of Zielona Góra

Szafrana $4 \mathrm{a}$

65-516 Zielona Góra

Poland

e-mail: M.Sudzik@wmie.uz.zgora.pl

Received: March 27, 2018

Revised: July 12, 2018 